\title{
Dimorphic Erythrocyte Population
}

National Cancer Institute

\section{Source}

National Cancer Institute. Dimorphic Erythrocyte Population. NCI Thesaurus. Code C117853.

The determination of the amount of dimorphic erythrocyes present in a sample. 